\title{
Patterns of indocyanine green videoangiography of choroidal tumours
}

\author{
Carol L Shields, Jerry A Shields, Patrick De Potter
}

\begin{abstract}
Background-Indocyanine green videoangiography is a recently popularised technique for imaging the retina and choroid. Little is known about the value of this technique in the evaluation of choroidal tumours.
\end{abstract}

Methods-Indocyanine green videoangiograms of 51 patients with choroidal tumours, including 32 with choroidal melanoma, five with choroidal naevus, four with choroidal metastasis, four with choroidal haemangioma, one with choroidal osteoma, one with choroidal vortex vein varix, and four with irradiated choroidal melanoma were reviewed in a masked fashion.

Results-The choroidal melanoma group achieved maximal fluorescence at an average of 18.2 (range 0.4 to 60 ) minutes after injection. At maximum fluorescence, the pattern varied from hypofluorescent in 17 cases, to isofluorescent in eight cases, and hyperfluorescent in seven cases. Non-pigmented choroidal melanoma generally showed an earlier average onset of fluorescence than the pigmented choroidal melanoma (mean 28 versus 185 seconds, respectively) $(p=0 \cdot 04)$. The two subgroups otherwise overlapped substantially in angiography characteristics. The choroidal metastasis group demonstrated maximal fluorescence at an average of 8.9 (range 1.7 to 13) minutes. All choroidal metastases had a homogeneous diffuse fluorescence with late isofluorescence. The choroidal metastases allowed a subtle blurred perception of the normal choroidal pattern through the tumour. The choroidal haemangioma group revealed very characteristic findings with an onset of filigree fluorescence at an average of 0.6 (range 0.4 to 0.7 ) minutes with little variation. The maximal fluorescence was typically hyperintense in all cases and was achieved at an average of 1.2 minutes and was reached by 1.8 minutes in all cases. In these cases the fluorescence appeared as a lacy diffuse 'fluorescent mulberry' pattern with visibility of the vascular channels and demonstrated 'washout' of the dye in the late frames.

Conclusion-Indocyanine green may be a useful adjunct in the differentiation of amelanotic choroidal melanoma, choroidal metastasis, and choroidal haemangioma. The different patterns of fluorescence may be explained by the intrinsic choroidal vascular architecture in these three tumours.

(Br F Ophthalmol 1995; 79: 237-245)

Indocyanine green is a tricarbocyanine dye that is more highly protein bound than sodium fluorescein dye. Therefore, it diffuses much more slowly out of fenestrated small choroidal vessels than does sodium fluorescein. These special properties of indocyanine green dye make it attractive as an agent to image the choroidal vasculature using fundus photography. Several recent reports have stressed the usefulness of this dye in the evaluation and treatment of choroidal neovascularisation. ${ }^{1-3}$

To our knowledge, there have been no clinical reports on the applicability of this dye in the diagnosis and management of a large series of choroidal tumours. In this report we detail the specific features of indocyanine green angiography in the evaluation of various choroidal tumours. We have identified specific circumstances where this new imaging method may prove useful in differentiating certain choroidal tumours, particularly amelanotic choroidal melanoma, choroidal metastasis, and choroidal haemangioma.

\section{Materials and methods}

Indocyanine green (Cardio-Green, Becton Dickinson Microbiology Systems, Cockeysville, MD, USA) was prepared in solution with the $25 \mathrm{mg}$ dose mixed in $2 \mathrm{ml}$ aqueous solvent and injected into a peripheral arm vein. A secondary saline bolus was not given after the original $2 \mathrm{ml}$ aqueous solvent. In all cases the injection site was the antecubital fossa or forearm venous system. Digital indocyanine green videoangiogram fundus photographs were then taken using a Topcon camera (TRC 50 IA) and printed on the Topcon IMAGEnet-H 1024 digital imaging system. The Topcon camera contained a halogen lamp of $300 \mathrm{~W}$ (a continuous light source) and an antireflection coating was applied to its optical system so that reflection of the near infrared spectral region was decreased. The exciter filter was on the side of the light source and the barrier filter on the side of the film.

The photographer complied with the following procedure: first photograph sooner than 10 seconds after injection, then photograph every 1 second up to 15 seconds after injection, then photograph every 5 to 10 seconds between 15 and 60 seconds, then photograph every 30 seconds between 1 and 3 minutes, then photograph every 60 seconds between 3 and 10 
Table 1 Patterns of indocyanine green fluorescence in 45 choroidal tumours

\begin{tabular}{|c|c|c|c|c|}
\hline & $\begin{array}{l}\text { Naevus } \\
(n=5)\end{array}$ & $\begin{array}{l}\text { Melanoma } \\
(n=32)\end{array}$ & $\begin{array}{l}\text { Metastasis } \\
(n=4)\end{array}$ & $\begin{array}{l}\text { Haemangioma } \\
(n=4)\end{array}$ \\
\hline \multicolumn{5}{|c|}{ Onset fluorescence (seconds): } \\
\hline Average & 36 & 134 & 30 & 33 \\
\hline Median & 26 & 40 & & \\
\hline Range & $17-59$ & $14-1228$ & $21-47$ & $26-43$ \\
\hline \multicolumn{5}{|l|}{ Pattern fluorescence: } \\
\hline Patchy & 0 & 3 & 0 & 0 \\
\hline Lacy & 4 & 13 & 0 & 4 \\
\hline Homogeneous & 1 & 15 & 4 & 0 \\
\hline Non-fluorescence & 0 & 1 & 0 & 0 \\
\hline \multicolumn{5}{|l|}{ Location fluorescence: } \\
\hline Focal & 0 & 12 & 0 & 0 \\
\hline Diffuse & 5 & 19 & 4 & 4 \\
\hline Non-fluorescence & 0 & 1 & 0 & 0 \\
\hline \multicolumn{5}{|c|}{ Choroidal vascular pattern: } \\
\hline Present & 4 & 21 & 4 & 0 \\
\hline Absent & 1 & 11 & 0 & $4^{\star}$ \\
\hline \multicolumn{5}{|c|}{ Maximum fluorescence (seconds): } \\
\hline Average & 709 & 1090 & 536 & 72 \\
\hline Median & 1130 & 1071 & 631 & 58 \\
\hline Range & $25-1139$ & $25-3600$ & $101-780$ & $49-110$ \\
\hline \multicolumn{5}{|c|}{ Intensity of fluorescence at maximum fluorescence: } \\
\hline Hypofluorescent & 3 & 17 & 0 & 0 \\
\hline Isofluorescent & 0 & 8 & 4 & 0 \\
\hline Hyperfluorescent & 2 & 7 & 0 & 4 \\
\hline \multicolumn{5}{|c|}{ Late hyperfluorescence ( $>30$ minutes): } \\
\hline Yes & 2 & 6 & 0 & 2 \\
\hline No & 3 & 26 & 4 & 2 \\
\hline
\end{tabular}

«The normal choroidal pattern was obliterated by the vascular pattern intrinsic to the tumour.

minutes, then five photographs between 10 and 35 minutes. The light intensity was adjusted as little as possible for late frames.

Digital indocyanine green angiography was performed in 51 patients with various choroidal tumours. All patients were examined by us and a detailed large fundus drawing was completed. Fundus photography, fluorescein angiography, and $A$ scan and $B$ scan ultrasonography were performed to confirm the diagnosis. ${ }^{4}$ The indocyanine green angiogram was performed subsequent to the above testing and at no cost to the patient.

The indocyanine green angiogram of the choroidal circulation was then analysed carefully in a masked fashion for earliest onset of fluorescence (in seconds), type of fluorescence pattern (patchy, lacy, homogeneous), location of the fluorescence (focal or diffuse), presence or absence of background choroidal vascular pattern in the region of the tumour, time of maximum fluorescence (in seconds), intensity

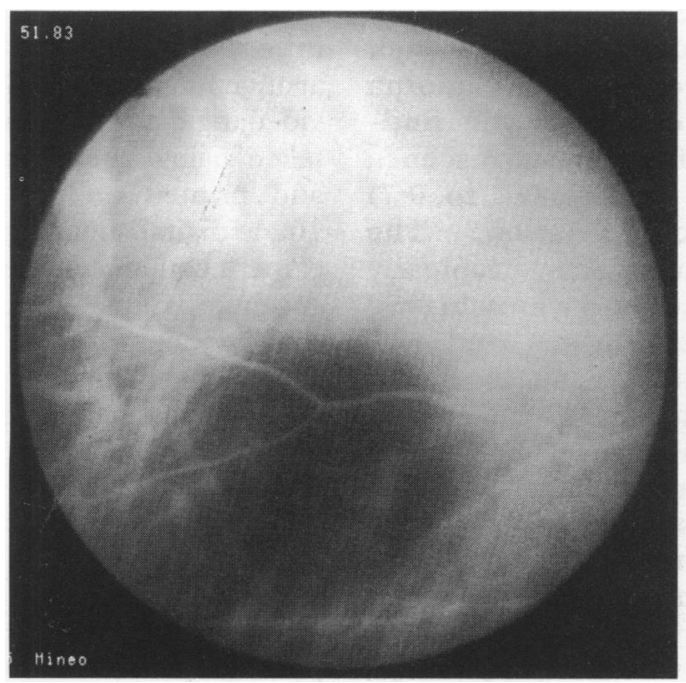

Fig $1 A$

Figure 1 Pigmented choroidal naevus. (A) Early frame at 51 seconds showing circumscribed hypofluorescence within the lesion. (B) Late frame at 40 minutes and 24 seconds showing persistent hypofluorescence. of maximum fluorescence (hyperfluorescent $4+$, hyperfluorescent 3+, hyperfluorescent $2+$, hyperfluorescent $1+$, isofluorescent, hypofluorescent 1+, hypofluorescent $2+$, hypofluorescent $3+$, hypofluorescent $4+$ ), and presence or absence of hyperfluorescence at more than 30 minutes after injection. The judgment of fluorescence intensity was relative to the surrounding 'normal' choroid. To avoid bias, the analysis of each angiogram was performed without details of the clinical findings.

The angiogram was then correlated with the clinical diagnosis and the findings were compared in a tabular fashion to assess for patterns of fluorescence in various tumours.

\section{Results}

We analysed 51 cases of choroidal tumours with indocyanine green videoangiography (Table 1). Of these 51 cases, 32 were choroidal melanoma (22 pigmented tumours and 10 non-pigmented tumours), five with choroidal naevus, four with choroidal metastasis, four with choroidal haemangioma, one with choroidal osteoma, one with vortex vein varix, and four with choroidal melanoma after plaque radiotherapy.

In all cases the angiogram was performed with sequential photography and the earliest frames ranged from 17 to 30 seconds while the latest frames were taken at a minimum of 31 minutes after injection. In all cases the normal choroidal pattern could be visualised around the edges of the choroidal tumour and the fluorescence patterns in the tumour were graded when compared with the surrounding normal choroid.

\section{CHOROIDAL NAEVUS}

We evaluated five cases of suspicious choroidal naevus with indocyanine green videoangiography (Table 1) (Figs 1, 2). The average time from dye injection to fluorescence was 36 seconds and the pattern of fluorescence was lacy diffuse in all but one case. The choroidal pattern was visible in four cases and obscured

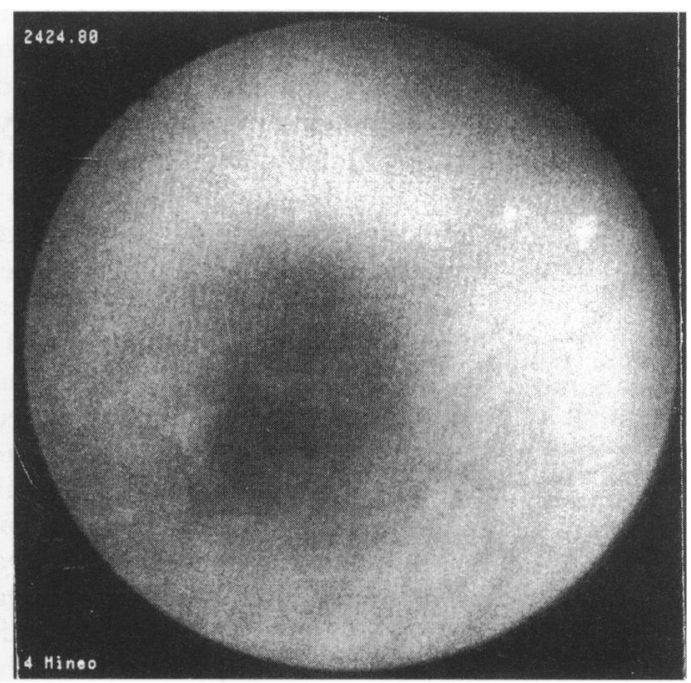

Fig $1 B$ 


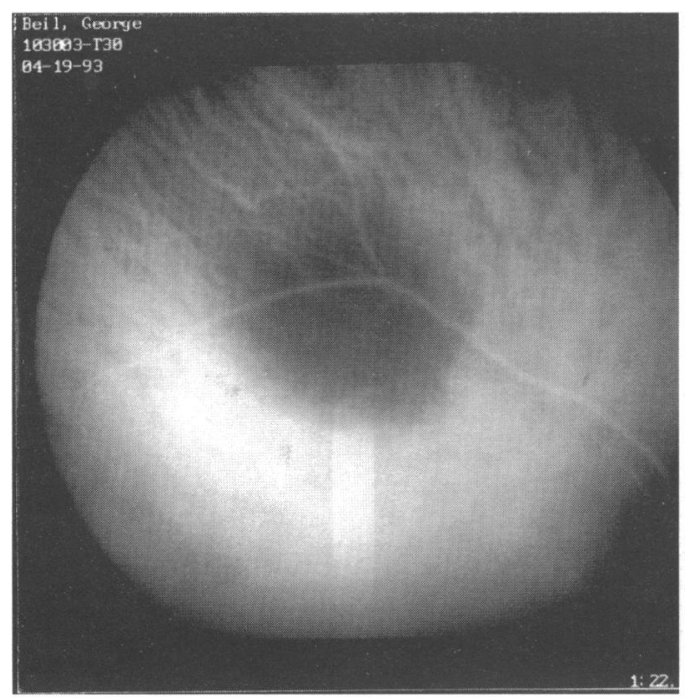

Fig $2 A$

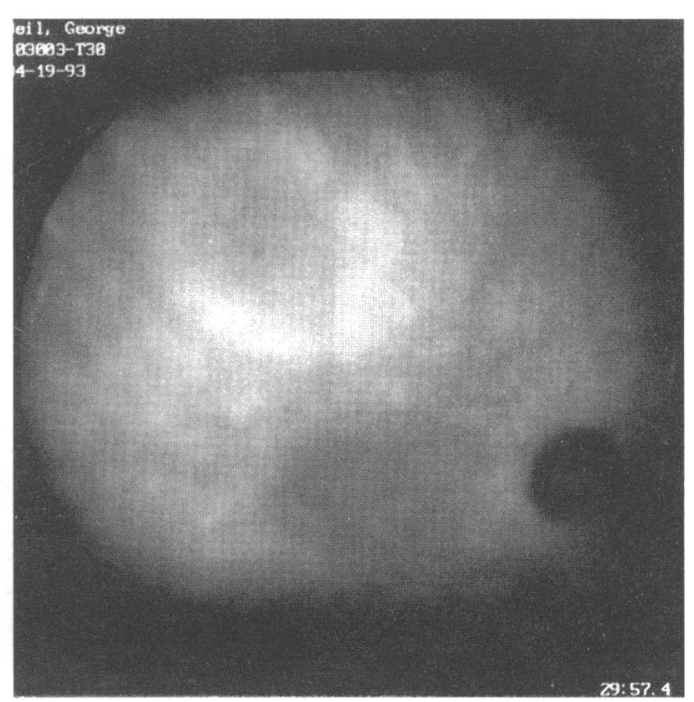

Fig $2 B$

Figure 2 Non-pigmented choroidal naevus. (A) Early frame at 82 seconds showing hypofluorescence within the lesion. (B) Late frame at 29 minutes and 57 seconds demonstrating central isofluorescence and a rim of hypofluorescence.

in one case of a non-pigmented suspicious choroidal naevus. Maximum fluorescence was achieved at an average of 709 seconds $(11.8$ minutes) and late fluorescence was present in two and absent in three cases.

\section{CHOROIDAL MELANOMA}

The patterns of indocyanine green videoangiography of choroidal melanoma were varied (Table 1) (Figs 3-6). The average time from dye injection to the onset of fluorescence was 134 seconds ( $2 \cdot 23$ minutes) but this was varied, ranging from 14 to 1228 seconds. Those tumours with delayed or minimal fluorescence tended to be darker in colour and more flat and infiltrative. Once fluorescence ensued, it developed one of several patterns: patchy in three cases; lacy with finer vasculature details in 13 cases; homogeneous without any vascular details in 15 cases; and absolutely no fluorescence in one flat infiltrative pigmented tumour. The fluorescence began as a

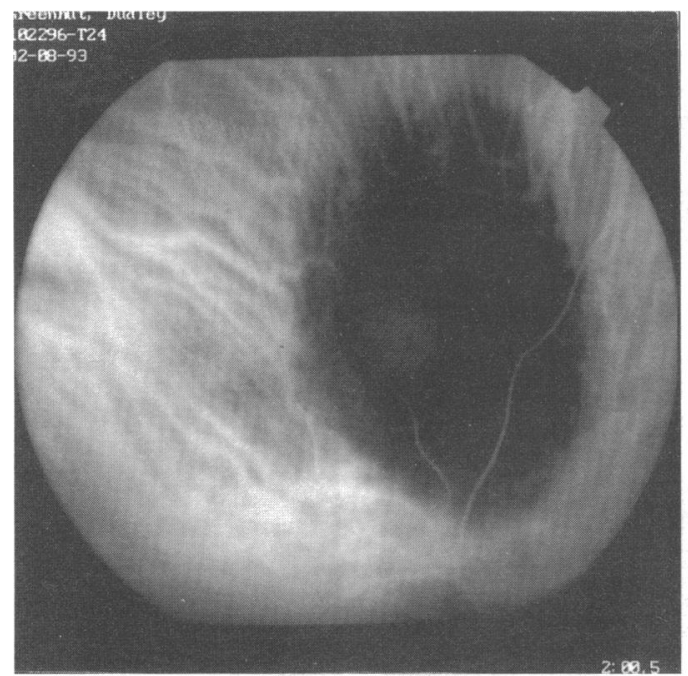

Fig $3 A$ focal area in 12 cases and as a diffuse area in 19 cases. The choroidal vascular pattern could be visualised in 21 of the 32 cases, but in most cases it was blurred and only partially visible. In 11 cases the choroidal vascular pattern was absent due to tumour presence. Maximum fluorescence was achieved at an average of 1090 seconds (18.2 minutes), but again this varied from 25 to 3600 seconds and was not dependent on the tumour thickness or colour. The intensity of the fluorescence compared with the surrounding normal choroid at each point in time was in general hypofluorescent. In eight cases it was isofluorescent and in seven it achieved hyperfluorescence. In the late frames ( $>30$ minutes) 26 tumours were hypofluorescent and six showed hyperfluorescence compared with the surrounding normal choroid.

A comparison of the 22 pigmented choroidal melanomas with the 10 non-pigmented choroidal melanomas showed that the indocyanine features overlapped substantially in

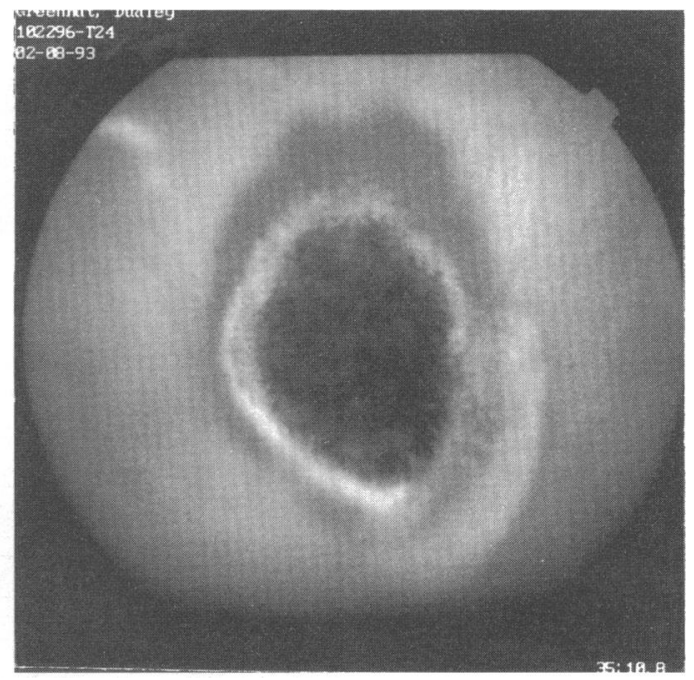

Fig $3 B$

Figure 3 Pigmented choroidal melanoma, dome shaped. (A) Early frame at 120 seconds showing dense central hypofluorescence within the tumour. (B) Late frame at 35 minutes and 10 seconds documenting a 'three ring pattern' with a persistent dense central hypofluorescence, a middle ring of hyperfluorescence, and a peripheral ring of relative hypofluorescence. Mild hyperfluorescence inferonasally represents subretinal fluid. 


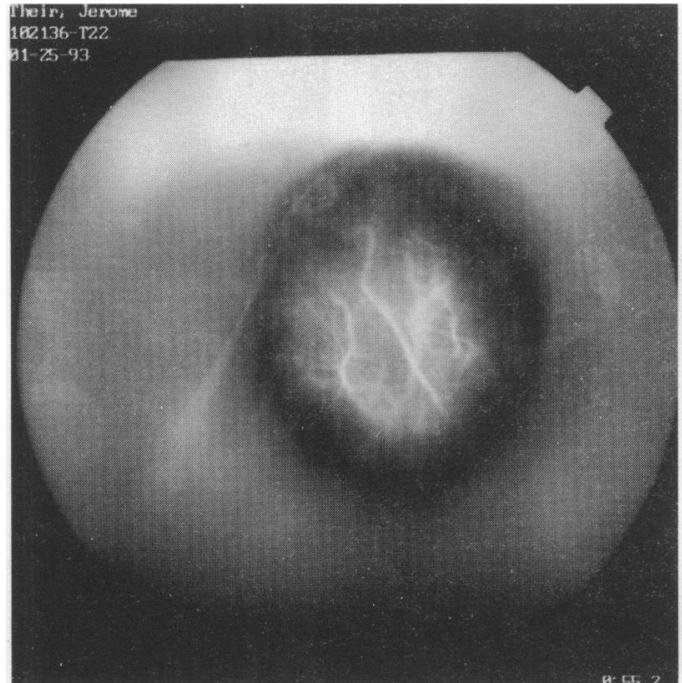

Fig $4 A$

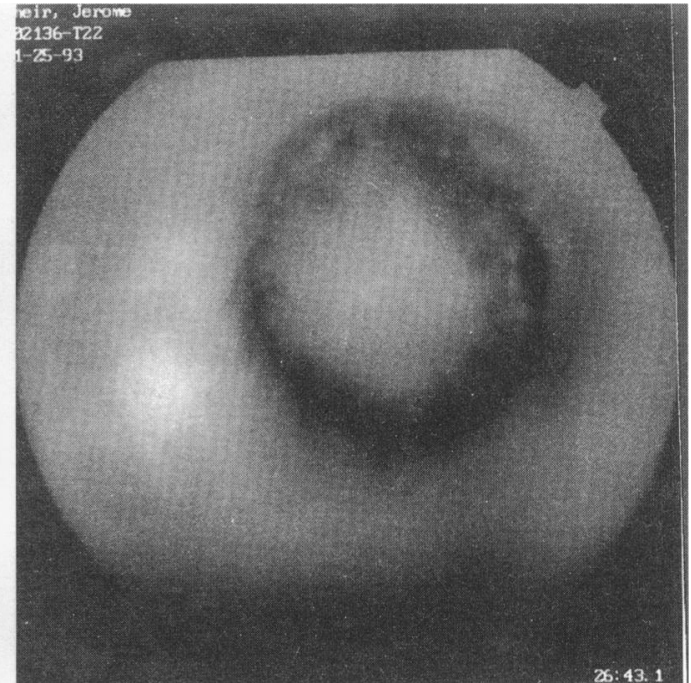

Fig $4 B$

Figure 4 Pigmented choroidal melanoma, mushroom shaped. (A) Early frame at 55 seconds showing prominent intratumoral vessels. (B) Late frame at 26 minutes and 43 seconds demonstrating isofluorescence centrally and hypofluorescence peripherally.

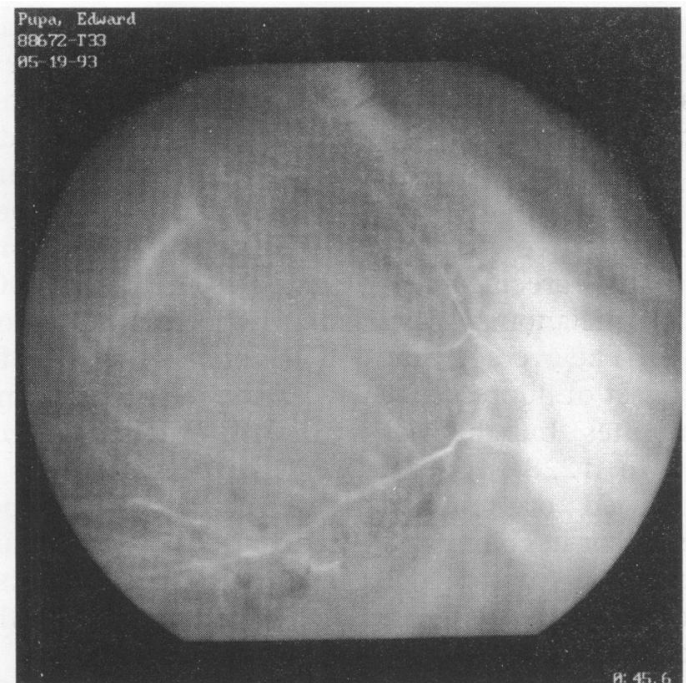

Fig $5 A$

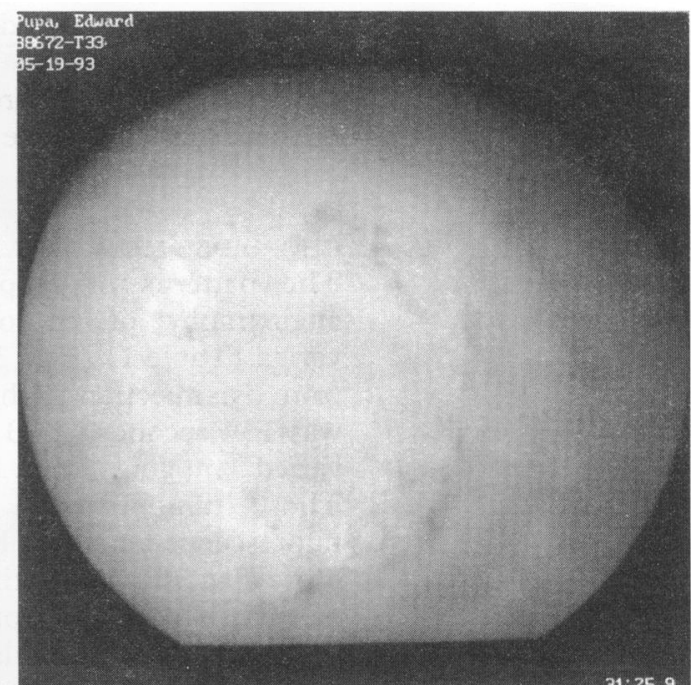

Fig $5 B$

Figure 5 Non-pigmented choroidal melanoma, dome shaped. (A) Early frame at 45 seconds showing visibility of the intrinsic tumour vessels contrasted with normal choroidal vessels. (B) Late frame at 31 minutes and 25 seconds demonstrating mild hyperfluorescence and spotty hyperfluorescent and hypofluorescent areas over the mass.

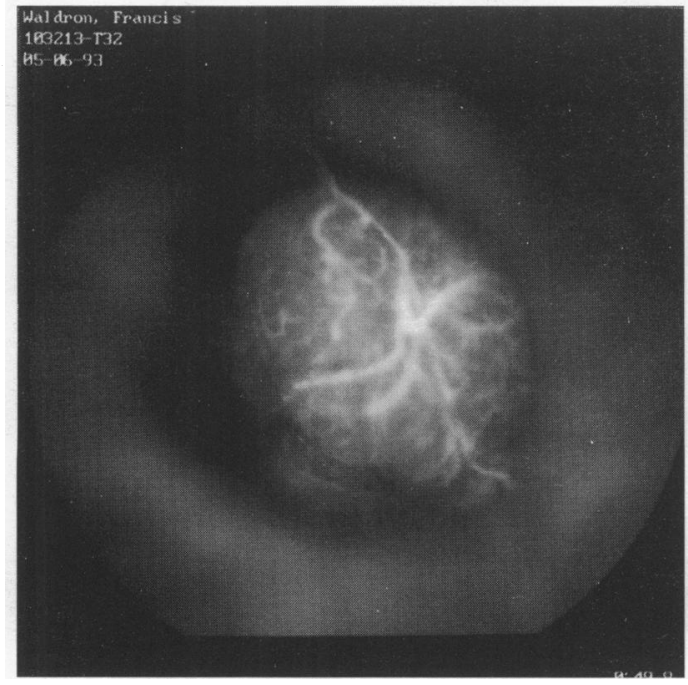

Fig 6 A

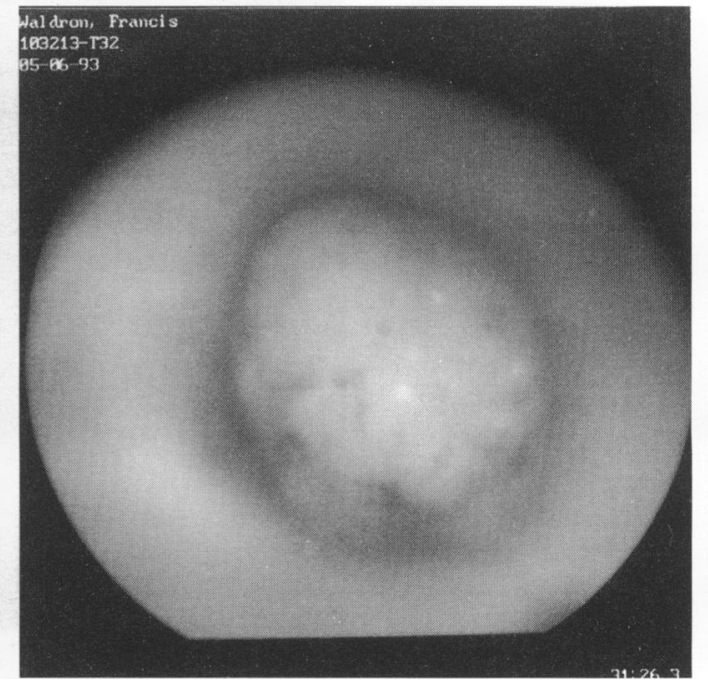

Fig $6 B$

Figure 6 Non-pigmented choroidal melanoma, mushroom shaped. (A) Early frame at 49 seconds demonstrating prominent intrinsic tumour vascularity. (B) Late frame at 31 minutes and 26 seconds showing relative isofuorescence. 
Table 2 Patterns of indocyanine green angiography in pigmented versus non-pigmented choroidal melanomas

\begin{tabular}{|c|c|c|}
\hline & \multicolumn{2}{|c|}{ Choroidal melanoma } \\
\hline & $\begin{array}{l}\text { Pigmented } \\
(n=22)\end{array}$ & $\begin{array}{l}\text { Non-pigmented } \\
(n=10)\end{array}$ \\
\hline \multicolumn{3}{|c|}{ Onset fluorescence (seconds): } \\
\hline Average & 185 & 28 \\
\hline Median & 47 & 25 \\
\hline Range & $14-1228$ & $17-40$ \\
\hline \multicolumn{3}{|l|}{ Pattern fluorescence: } \\
\hline Patchy & 3 & 0 \\
\hline Lacy & 11 & 2 \\
\hline Homogeneous & 7 & 8 \\
\hline Non-fluorescence & 1 & 0 \\
\hline \multicolumn{3}{|c|}{ Location fluorescence: } \\
\hline Focal & 11 & 1 \\
\hline Diffuse & 10 & 9 \\
\hline Non-fluorescence & 1 & 0 \\
\hline \multicolumn{3}{|c|}{ Choroidal vascular pattern: } \\
\hline $\begin{array}{l}\text { Present } \\
\text { Absent }\end{array}$ & $\begin{array}{r}13 \\
9\end{array}$ & 8 \\
\hline \multicolumn{3}{|c|}{ Maximum fluorescence (seconds): } \\
\hline Average & 1115 & 1038 \\
\hline Median & 1130 & 1035 \\
\hline Range & $69-3600$ & $25-2299$ \\
\hline \multicolumn{3}{|c|}{ Intensity fluorescence: } \\
\hline Hypofluorescent & 14 & 3 \\
\hline Isofluorescent & 4 & 4 \\
\hline Hyperfluorescent & 4 & 3 \\
\hline \multicolumn{3}{|c|}{ Late hyperfluorescence ( $>30$ minutes): } \\
\hline Yes & 2 & 4 \\
\hline No & 20 & 6 \\
\hline
\end{tabular}

these small groups (Table 2). The nonpigmented tumours had a more homogeneous pattern than the pigmented tumours. The intensity of fluorescence was judged less in the pigmented tumour group with $64 \%$ being hypofluorescent while $30 \%$ of the nonpigmented tumours were hypofluorescent. Statistical analysis using the unpaired $t$ test showed that the non-pigmented choroidal melanomas had a significantly earlier onset of fluorescence than the pigmented tumours [ $t(\mathrm{df} 24 \cdot 1)=2 \cdot 17 ; \mathrm{p}=0.04]$. The time of maximum fluorescence did not differ significantly between the two subgroups $[t$ (df $18 \cdot 8)=0.08 ; p=0.94]$.

CHOROIDAL METASTASIS

There were four cases of choroidal metastasis:

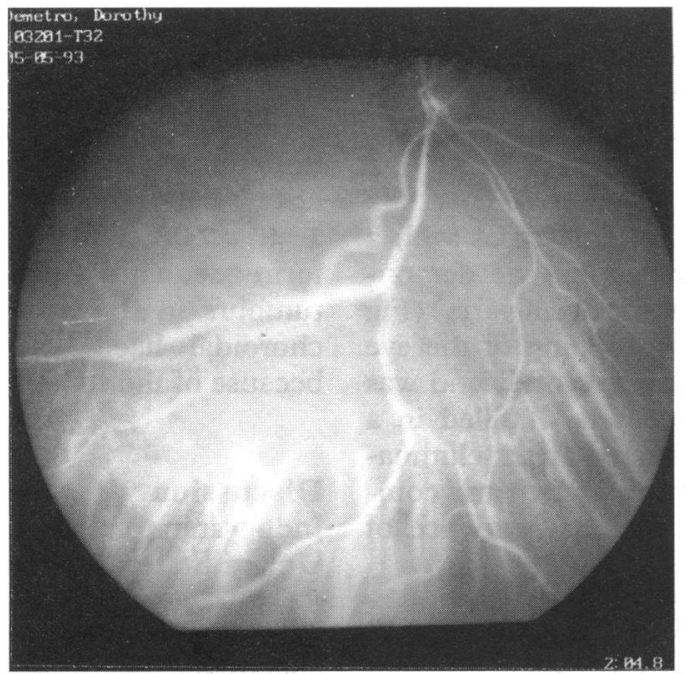

Fig $7 A$ three originating from breast carcinoma and one from renal cell carcinoma (Table 1) (Fig 7). The onset of fluorescence averaged 30 seconds and all four cases showed a similar pattern of subtle diffuse homogeneous fluorescence of the tumour. A blurred choroidal pattern was visible in all four cases in the region of the tumour. In one case the normal choroidal pattern abruptly and dramatically diminished in the region of the tumour. All four tumours showed ill defined isofluorescence without hyperfluorescence and in the late frames they were hypofluorescent overall. The three cases of breast carcinoma metastases were remarkably similar in their angiogram characteristics.

\section{CHOROIDAL HAEMANGIOMA}

There were four cases of circumscribed choroidal haemangioma (Table 1, Fig 8). All four cases had similar findings with little variability. The onset of fluorescence occurred at 33 seconds on average and achieved a maximum rapidly by an average of 72 seconds. This was substantially more rapid than all other tumours in this study. The fluorescence typically occurred as a lacy diffuse hyperintense pattern with filling of the peripheral portion of the haemangioma first then the central portion as imaged with rapid sequence photography. The normal choroidal pattern was obscured in all cases by the vascular channels of the tumour. The fluorescence cleared at variable rates leaving traces of fluorescence in the vascular channels giving the silhouettes of the vascular channels. In the late frames in two cases, the tumour was hypofluorescent as if the dye had 'washed out'. In all cases, a rim of hyperfluorescence surrounding the tumour was seen in the late frames.

CHOROIDAL OSTEOMA

We evaluated one case of choroidal osteoma

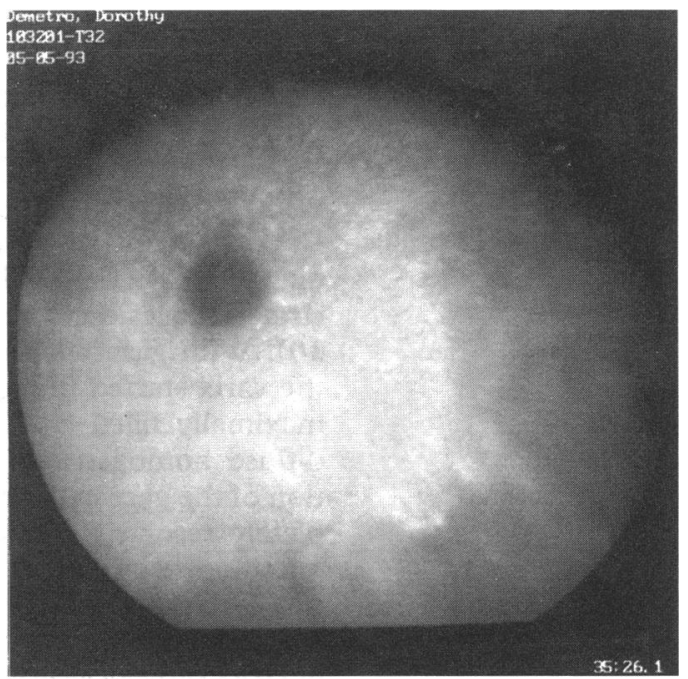

Fig $7 B$

Figure 7 Choroidal metastasis. (A) Early frame at 124 seconds showing smudgy isofluorescence of the mass surrounding the optic disc and within the macula. Note the normal choroidal pattern abruptly stops at the margin of the metastasis and is barely visible within the mass. (B) Late frame at 35 minutes and 26 seconds demonstrating overall isofluorescence with overlying diffuse spotty hyperfluorescence. 


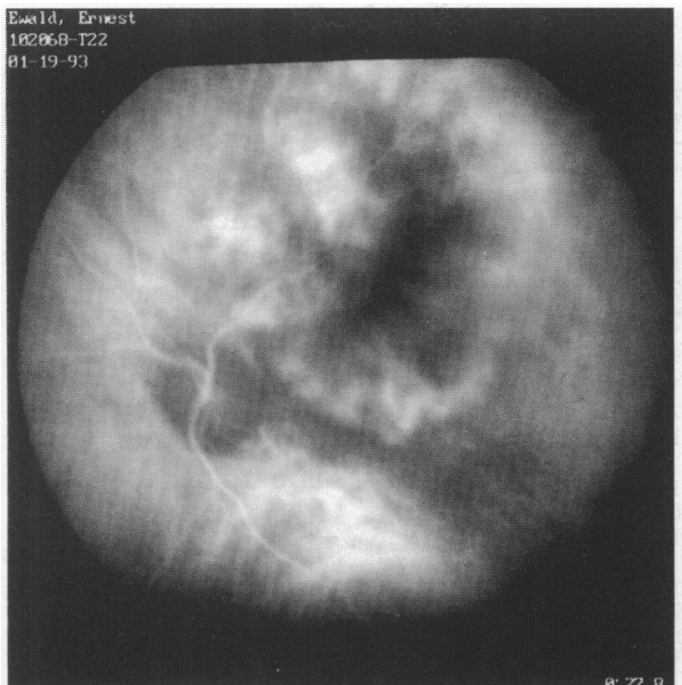

Fig $8 A$

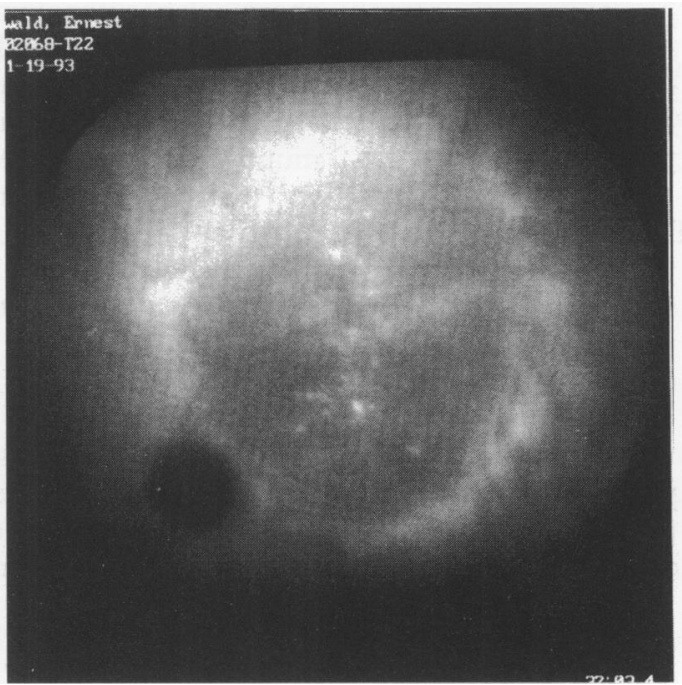

Fig $8 C$

with indocyanine green videoangiography (Fig 9). The onset of tumour fluorescence occurred at 92 seconds and was diffuse and homogeneous. The choroidal vascular pattern could be visualised through the tumour. Maximal fluorescence was achieved at 562 seconds and overall the tumour remained hypofluorescent to the surrounding choroid.

\section{CHOROIDAL VORTEX VEIN VARIX}

In one patient who was referred to us with a diagnosis of choroidal melanoma we demonstrated the lesion to be a vortex vein varix ${ }^{5}$ (Fig 10). With gaze evoked positioning of the eye the varix started filling at 38 seconds and was maximally filled at 70 seconds. It filled in a diffuse homogeneous pattern. After elimination of the gaze evoked stimulus the varix completely resolved without residual evidence of indocyanine green.

\section{IRRADIATED CHOROIDAL MELANOMA}

There were four cases of iodine-125 plaque irradiated choroidal melanoma in this evaluation. All four cases were within 4 months of irradiation. The fluorescence patterns showed

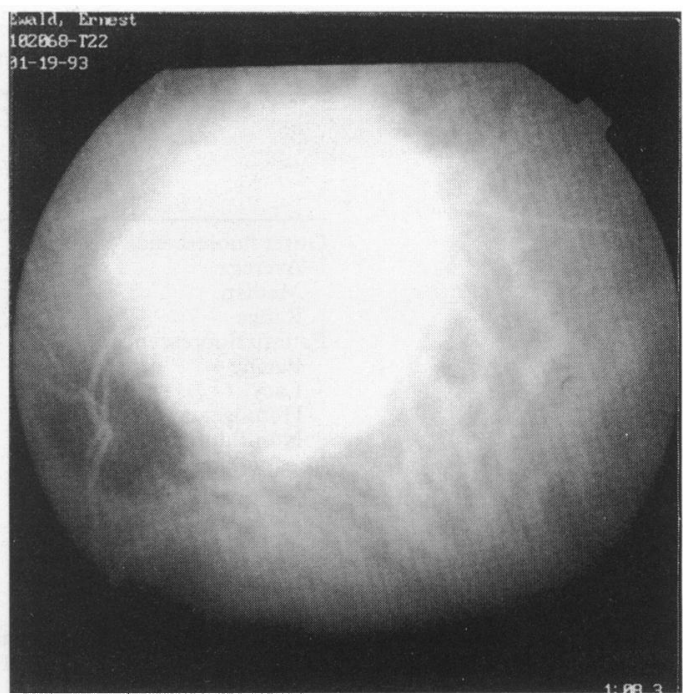

Fig $8 B$

Figure 8 Choroidal haemangioma. (A) Very early frame at 27 seconds showing a filigree of tumour vessels filling with dye at the margin of the mass. (B) Early frame at 68 seconds documenting the typical, brilliant rapid

hyperfluorescence obscuring the choroidal pattern. (C) Late frame at 32 minutes and 3 seconds demonstrating the 'washout' phase with central hypofluorescence and a rim of staining outside the margins of the tumour.

onset of fluorescence in the region of the tumour at $63,795,28$ seconds respectively and in one case there was no fluorescence at all. The fluorescence was diffuse and the intensity was isofluorescent in two cases and hypofluorescent in two cases. In two cases we compared preirradiation versus post-irradiation angiograms and there was a decrease in the fluorescence onset and intensity after irradiation in both cases.

STATISTICAL ANALYSIS

A statistical analysis was performed on the choroidal melanoma group comparing the two subgroups (pigmented versus non-pigmented choroidal melanoma) with regard to the time of onset of fluorescence and the time of maximal fluorescence. Results of the $t$ test revealed that non-pigmented choroidal melanoma showed significantly earlier onset of fluorescence $[t(\mathrm{df} 24 \cdot 1)=2 \cdot 17 ; \mathrm{p}=0 \cdot 04]$ than pigmented choroidal melanoma. Time of maximal fluorescence showed no statistical difference between the two groups $[t$ (df $18 \cdot 8)=0.08 ; p=0.94]$. We were unable to perform adequate statistical analysis on the other tumours in this study (choroidal metastasis, choroidal haemangioma, choroidal naevus) because of the small sample size.

\section{Discussion}

Indocyanine green is a dye approved by the United States Food and Drug Administration for intravenous use in cardiac, hepatic, and ophthalmic imaging studies. ${ }^{1}$ Kogure and coworkers first used this dye in 1970 as an intra-arterial injection to study choroidal absorption angiography in monkeys. ${ }^{6}$ David was the first to use intra-arterial indocyanine green angiography of the choroidal circulation 


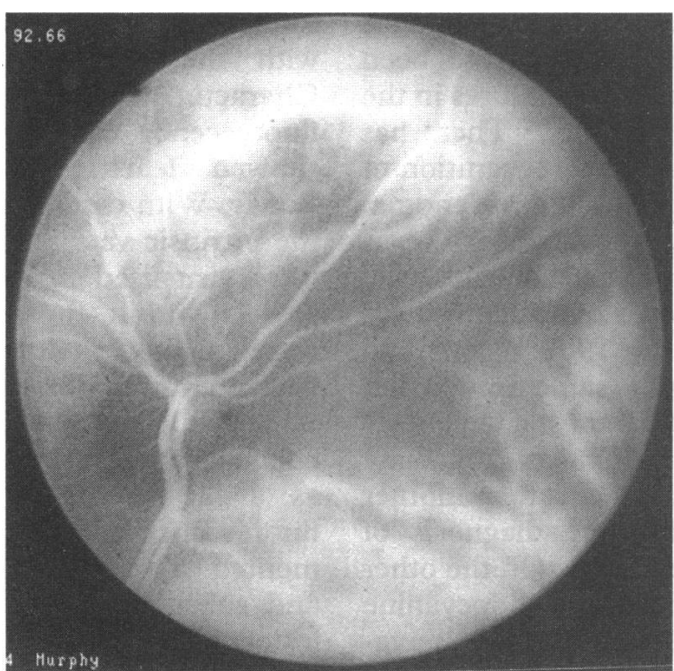

Fig $9 A$

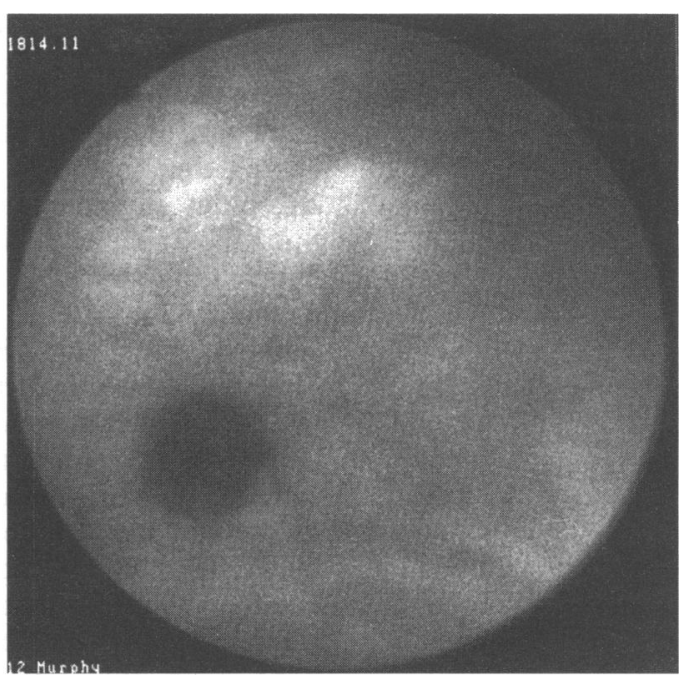

Fig $9 B$

Figure 9 Choroidal osteoma. (A) Early frame at 92 seconds showing relative hypofluorescence within the mass. (B) Late frame at 30 minutes and 14 seconds demonstrating isofluorescence.

in human patients. ${ }^{7}$ Hochheimer then reported on the use of infrared angiography of the cat fundus using an intravenous not an intraarterial injection of indocyanine green. ${ }^{8}$ Flower and Hochheimer ${ }^{9-11}$ then studied intravenous indocyanine green angiography in humans in the early 1970s and, later, Bischoff and Flower $^{12}$ reported on 10 years' experience with 500 choroidal angiograms of various fundus disease.

It has been more than 20 years since the initial investigations of indocyanine green angiography of the choroidal circulation. Advances in infrared imaging technology have improved the resolution of the choroidal angiograms tremendously and new enthusiasm has emerged for the use of this dye. Recent reports have focused on the usefulness of indocyanine green in identifying choroidal neovascularisation, especially those which are poorly defined on fluorescein angiography. ${ }^{1-3}$

The findings of indocyanine green angiography of choroidal tumours were superficially described in the early developmental years of the

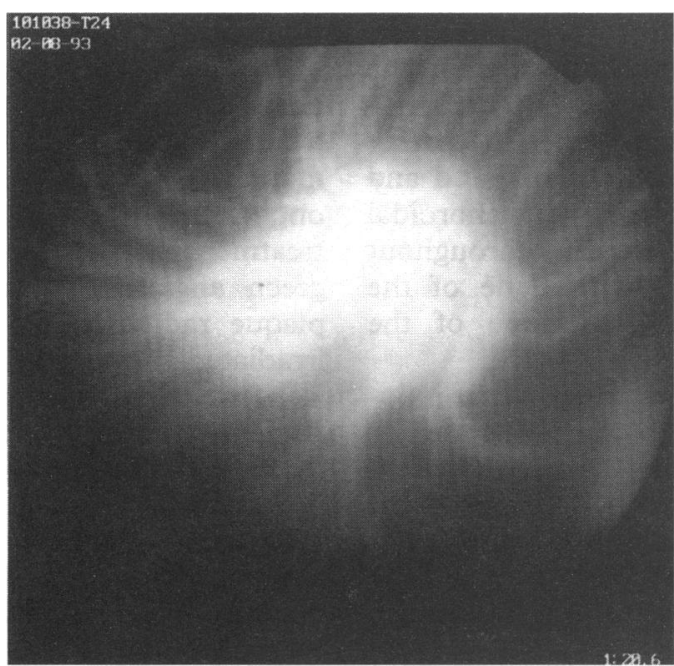

Fig $10 A$ dye technology. ${ }^{12-17}$ These authors found that the information on choroidal tumours derived by using indocyanine green was twofold: the vascularisation of non-pigmented tumours could be studied and the angiogram could provide a topographic parameter for delineating the size and growth of choroidal tumours. Owing to the deficient resolution of the early imaging systems, details of pigmented choroidal tumours (choroidal naevus and melanoma) were not clearly imaged and the few tumours that were imaged were found to absorb the infrared light leaving a shadow. The early limited reports of non-pigmented choroidal tumours showed various patterns and the few cases of choroidal haemangioma were found to be highly fluorescent. ${ }^{1416}$ Our experience with the new technology of indocyanine green videoangiography will add to these early observations.

Most intraocular tumours are best recognised and differentiated using indirect ophthalmoscopy. ${ }^{4}$ In difficult cases, standard fluorescein angiography, ocular ultrasonography, radioactive phosphorus $\left({ }^{32} \mathrm{P}\right)$ uptake

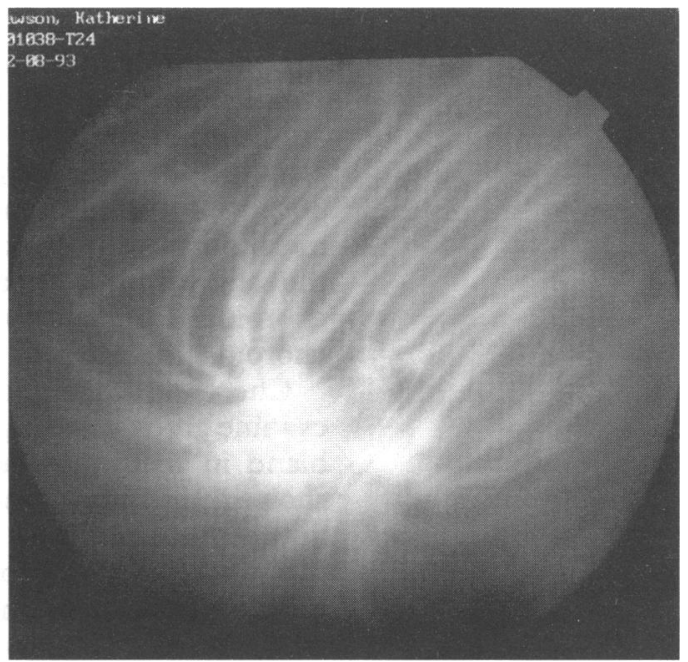

Fig $10 B$

Figure 10 Choroidal vortex varix. (A) Varix fully filled and hyperfluorescent after valsalva manoeuvre. (B)Varix deflated showing the relatively normal choroidal pattern except at the vortex ampulla where minimal dilatation persists. 
testing, contrast enhanced magnetic resonance imaging, and fine needle biopsy have been shown to be helpful adjunctive measures in the diagnosis of intraocular tumours. ${ }^{4}$ There has been a trend towards improved recognition of choroidal melanoma and pseudomelanoma. Studies from pathology specimens of eyes enucleated with the presumed diagnosis of uveal melanoma have shown that the misdiagnosis rate was $20 \%$ in the $1960 \mathrm{~s}^{18}$ and had improved to $1.9 \%$ during the same time period at a centre with advanced diagnostic facilities ${ }^{19}$ and later improved to $0.75 \%$ at this same centre. ${ }^{20}$ For this reason, it seems that another diagnostic test to confirm the diagnosis of uveal melanoma is not necessary. On the other hand, we suspect that the use of indocyanine green videoangiography, based on the observations from this report, may provide useful information in the differentiation of amelanotic choroidal tumours such as choroidal amelanotic melanoma, haemangioma, and metastasis. These amelanotic choroidal tumours are often the most difficult to differentiate from one another and their choroidal architecture as visualised by indocyanine green dye may be helpful.

In this report, the indocyanine green fluorescence findings for choroidal melanoma were the most varied of all the uveal tumours. An increasing degree of pigmentation of the tumour was associated with less fluorescence. In one case of a deeply pigmented diffuse choroidal melanoma there was complete nonfluorescence throughout the entire angiogram while the surrounding choroid showed normal fluorescence. In most cases, the seemingly normal choroidal pattern could be visualised either at the rim of the tumour or within the tumour itself. Early fluorescence was usually seen within the first 1 to 2 minutes, but in some cases it was difficult to differentiate tumour fluorescence from the normal underlying choroidal fluorescence. The choroidal melanoma group achieved maximal fluorescence at an average of 1090 seconds (18.2 minutes) but again this was quite variable. The only feature that seemed to differentiate choroidal melanoma and naevus from the other tumours was that they were more likely to be relatively hypofluorescent throughout the entire angiogram with respect to the surrounding normal choroid than were the other tumours. In fact, $64 \%$ of the pigmented and $30 \%$ of the non-pigmented choroidal melanomas were hypofluorescent throughout the angiogram compared with none of the choroidal haemangiomas and none of the choroidal metastases.

Choroidal metastases imaged with indocyanine green videoangiography were generally bland in their vascular appearance. They all showed isofluorescence at their brightest fluorescence and in all cases a blurred image of the underlying choroidal pattern could be visualised. Both of these features were seen with choroidal melanoma and none of these features were seen with choroidal haemangioma.

Perhaps the most dramatic fluorescence on indocyanine green videoangiography was seen with the choroidal haemangioma group. Characteristically, they all achieved maximal fluorescence by nearly 1 minute and then showed clearance of the fluorescence more slowly. With careful inspection, the details of the intrinsic vascular channels of the tumour were visualised in these cases. The tumour vessels within the choroidal haemangioma were distinctly different from those seen within choroidal melanoma and those of the underlying normal choroidal pattern. The choroidal haemangioma vascularity appeared as a lacy compact curvilinear 'mulberry' fluorescence and in three cases it was documented to originate at the margin of the lesion and subsequently involve the centre of the lesion. The tumour was always brilliantly hyperintense in its fully filled stage and demonstrated a 'washout' of dye in the late frames. In contrast, the intrinsic vascularity of choroidal melanoma involved larger calibre vessels with a branching elongated pattern quite similar to that seen on fluorescein angiography and none showed the dramatic rapid hyperfluorescence and subsequent 'washout' hypofluorescence seen with haemangioma. Similarly, the normal choroidal pattern showed elongated large calibre vessels in an elegant flowing fashion quite unlike that seen with choroidal haemangioma.

Our preliminary studies suggest that indocyanine green videoangiography may be of some assistance in the differential diagnosis of amelanotic choroidal tumours. The patterns of fluorescence vary with the different tumours but it is most predictable with choroidal haemangioma. In the cases of amelanotic choroidal tumours it is essential to obtain early frames within a few seconds of intravenous injection of the dye to assess for a choroidal haemangioma pattern. The late frames are also helpful, as choroidal metastases may be isofluorescent and melanoma hypofluorescent or variable.

Indocyanine green videoangiography may also be useful in evaluating for radiation induced ischaemia of a plaque irradiated tumour. We have documented this in two cases in this report, but we caution that it may be difficult to differentiate choroidal ischaemia from choroidal pigment in the heavily pigmented tumours. We have not yet had the opportunity to compare a large group of pretreatment with post-treatment indocyanine green angiography in patients managed by plaque radiotherapy. We speculate that the irradiated tumours will show more choroidal ischaemia, but longer follow up with repeat angiograms will be necessary to evaluate the degree and significance of the choroidal ischaemia.

Several limitations of this investigation are realised and include the lack of correlation of the injection dilution with patient weight, lack of calculation of time from injection in the forearm to the choroid (arm to choroid time), and lack of inclusion of overall patient systemic vascular status. These factors could all 
contribute to variations in the time of onset, time of maximal fluorescence, and subjective intensity interpretations. Unfortunately, the standard recommended technique of dye injection from the Cardio-Green package insert does not include calculation and dilution on a weight basis. The Cardio-Green recommended technique, which we employ in our photography department, is stated to provide optimal concentration of the dye bolus to image the choroidal vasculature. In this report we recorded the time at the various stages of dye flow to create objective data for interpretation. Perhaps the specific time in seconds should not be interpreted as specific times necessary to make a diagnosis, but only as general time guidelines useful for assisting in a diagnosis.

Indocyanine green videoangiography is still in its definition phase. Although most choroidal tumours are diagnosed by indirect ophthalmoscopy and more traditional ancillary studies, this adjunctive test may prove useful in difficult cases, especially amelanotic choroidal tumours. We realise that it is difficult to assign exact patterns or specific times for fundus angiography of any type, but the purpose of this investigation was to evaluate the indocyanine green angiographic characteristics of various choroidal tumours. Better imaging modalities with improved resolution capabilities will be helpful to define more exact fluorescence patterns. The future of indocyanine green angiography in the diagnosis, treatment, and follow up of choroidal tumours is still evolving.

Support was provided by the Macula Foundation, New York, NY and the Eye Tumor Research Foundation, Philadelphia, USA.

Statistical analysis was provided by Jacqueline Cater, $\mathrm{PhD}$, Department of Biostatistics and Academic Computing Thomas Jefferson University, Philadelphia, PA, USA.
1 Destro M, Puliafito CA. Indocyanine green videoangiography of choroidal neovascularization. Ophthalmology 1989; 96: 846-53.

2 Guyer DR, Puliafito CA, Mones JM, Friedman E, Chang W, Verdooner SR. Digital indocyanine green angiography in chorioretinal disorders. Ophthalmology 1992; 99: 287-91.

3 Yanuzzi LA, Slakter JS, Sorenson JA, Guyer DR, Orlock DA. Digital indocyanine green videoangiography and

4 Shields JA, Shields CL. Intraocular tumours: $a$ text and atlas. Philadelphia: WB Saunders, 1992: 117-36.

5 Singh AD, De Potter P, Shields CL, Shields JA. Indocyanine green angiography and ultrasonography of a varix of the green angiography and ultrasonography of a var
vortex vein. Arch Ophthalmol 1993; 100: 1283-4

6 Kogure K, David NJ, Yamanouchi U, Choromokos E. Infrared absorption angiography of the fundus circulation. Arch Ophthalmol 1970; 83: 209-14.

7 David NJ. Infra-red absorption fundus angiography. In: Amalric P, ed. Fluorescein angiography: Proc Int Symp 1969. Basel: Karger, 1971: 189-92.

8 Hochheimer BF. Angiography of the retina with indocyanine green. Arch Ophthalmol 1971; 86: 564-5.

9 Flower RW, Hochheimer BF. Clinical infrared absorption angiography of the choroid [Letter]. Am $\mathcal{f}$ Ophthalmol 1972; 73: 458-9.

10 Flower RW, Hochheimer BF. A clinical technique and apparatus for simultaneous angiography of the separate retinal and choroidal circulations. Invest Ophthalmol 1973 12: $248-61$.

11 Flower RW. Infrared absorption angiography of the choroid and some observations of the effects of high intraocular pressures. Am f Ophthalmol 1972; 74: 600-14.

12 Bischoff PM, Flower RW. Ten years experience with choroidal angiography using indocyanine green dye: a new routine examination or an epilogue? Doc Ophthalmol 1985; 60: 235-91.

13 Patz A, Flower RW, Klein ML, Orth DH, Fleischman JA, McLeod S. Clinical application of indocyanine green angiography. In: De Laey JJ, ed. International symposium angiography. In: De Laey JJ, ed. International symposium
on fluorescein angiography, 1976. The Hague: Dr W Junk, 1976: 245-51. (Doc Ophthalmol Proc Ser; 9).

14 Habozit F. Angiographie choroidienne au vert d'indocyanine. These Lyon Med 1976.

15 Francois P, Turut P, Delannoy C. L'angiofluorographie choroidienne a l'indocyanine. Bull Soc Ophtalmol Fr 1977 77: 971-2.

16 Chopdar A, Turk AM, Hill DW. Fluorescent infra-red angiography of the fundus oculi using indocyanine green dye. Trans Ophthalmol Soc UK 1978; 98: 142-6.

17 Bacin F, Buffet J-M, Mutel N. Angiographie par absorption, en infrarogue, au vertd, indocyanine. Aspects chez le sujet normal et dans les tumeurs choroidiennes. Bull Soc Ophtalmol Fr 1981; 81: 315-9.

18 Shields JA, Zimmerman LE. Lesions simulating malignan melanoma of the posterior uvea. Arch Ophthalmol 1973 89: 466-71.

19 Shields JA, McDonald PR. Improvements in the diagnosis of posterior uveal melanomas. Arch Ophthalmol 1974; 91: 259-64.

20 Shields JA, Shields CL. Intraocular tumors: $a$ text and atlas. Philadelphia: WB Saunders, 1992: 137-9. 